\title{
HÁBITOS ALIMENTICIOS DEL GÉNERO GYRIOSOMUS GUÉRIN-MÉNEVILLE, 1834 (COLEOPTERA: TENEBRIONIDAE): ¿QUÉ COMEN LAS VAQUITAS DEL DESIERTO COSTERO?
}

\author{
FOOD HABITS OF THE GENUS GYRIOSOMUS GUÉRIN-MÉNEVILLE, 1834 \\ (COLEOPTERA: TENEBRIONIDAE): WHAT DO THE VAQUITAS OF THE \\ COASTAL DESERT EAT?
}

\author{
Jaime Pizarro-Araya ${ }^{1}$
}

\begin{abstract}
RESUMEN
En el presente trabajo se describen por primera vez los hábitos alimenticios y modificaciones en la ecología trófica de algunas especies del género Gyriosomus (Coleoptera: Tenebrionidae). Se discute respecto a las posibles causas de estas adaptaciones tróficas y sus posibles implicancias ecológicas.

Palabras clave: Gyriosomus, Tenebrionidae, desierto costero, ecología trófica.
\end{abstract}

\section{ABSTRACT}

In the present work the food habits and the changes in the trophic ecology of some species of the genus Gyriosomus (Coleoptera: Tenebrionidae) are described for the first time, and the possible causes of these trophic adaptations and their ecological implications are discussed.

Key words: Gyriosomus, Tenebrionidae, coastal desert, trophic ecology.

Gyriosomus Guérin-Méneville 1834, con 37 especies descritas a la fecha (Pizarro-Araya y Flores, 2006; Pizarro-Araya et al., 2008), es uno de los géneros endémicos y erémicos más diversificado en Chile. Pertenece a la tribu Nycteliini, un taxón endémico de América del Sur (Flores, 2000). Las especies de Gyriosomus se hallan distribuidas desde la Reserva Nacional Paposo ( $25^{\circ} 05^{\prime} \mathrm{S}$ y $70^{\circ} 29^{\prime} \mathrm{O}$, Región de Antofagasta) hasta la precordillera de Rancagua (34 $11^{\prime}$ 'S y $70^{\circ} 39^{\prime} \mathrm{O}$, Región de O'Higgins) (Pizarro-Araya y Jerez, 2004), áreas pertenecientes a las provincias biogeográficas de Coquimbo y Santiago de la subregión Chilena Central (sensu Morrone, 2006).

Las especies de Gyriosomus son diurnas, epígeas y están asociadas principalmente con la vegetación arbustiva y herbácea (perennes y anuales) de terrazas costeras, planicies y cuencas de la Depresión Intermedia (Pizarro-Araya y Flores, 2004; Alfaro et al., en prensa). En su distribución austral, los adultos se encuentran en cerros bajos con escasa cubierta vegetal, como es el caso de Gyriosomus laevigatus Guérin-Méneville, 1834 (Sáiz et al., 1989).

Las especies pertenecientes al género Gyriosomus han sido descritas inicialmente como fitófagas (Gebien, 1944; Peña, 1959), alimentándose de plantas rastreras, especialmente de pétalos y partes blandas (Vidal y Guerrero, 2007). Al respecto, Mondaca (2004) señala que la alimentación de los adultos se compone de tejidos vegetales subterráneos de plantas anuales y perennes. De manera similar, PizarroAraya y Flores (2004) documentan que Gyriosomus granulipennis (Pizarro-Araya y Flores, 2004) se

\footnotetext{
1 Laboratorio de Entomología Ecológica, Departamento de Biología, Facultad de Ciencias, Universidad de La Serena; Casilla 599, La Serena, Chile. E-mail: japizarro@userena.cl
} 
alimenta de flores y hojas de Frankenia chilensis K. Presl (Frankeniaceae) y Nolana sp. (Nolanaceae), mientras que Gyriosomus multigranulosus PizarroAraya y Flores, 2004, forrajea hojas de Frankenia chilensis, Nolana brunonianus Hook. et. Arn, Nolana sedifolia Poepp. y Haplopappus foliosus DC. (Asteraceae), todas especies asociadas al matorral estepario costero (sensu Gajardo, 1993). Además, Cepeda-Pizarro et al. (2005a), al estudiar las comunidades de artrópodos epígeos del desierto costero transicional chileno $\left(25-32^{\circ} \mathrm{S}\right)$, reportan que algunas especies vegetales de geófitas y anuales (e.g., Rhodophiala bagnoldii [Herb.] Traub) son forrajeadas intensamente por los adultos del género Gyriosomus (Figura 1), lo que concuerda con la fenología de las plantas y con una mayor disponibilidad del recurso alimenticio (Armesto et al., 1993).

La ecología trófica de Gyriosomus, como ocurre con la gran mayoría de los tenebriónidos endémicos de Chile, es casi por completo desconocida. En este contexto, el objetivo del presente trabajo es documentar los hábitos alimenticios y los cambios en la ecología trófica de ciertas especies de Gyriosomus presentes en el desierto costero chileno y, junto con ello, discutir la relevancia que este género tiene dentro de la trama trófica, al generar diferentes tipos de interacciones ecológicas en los sistemas de desierto.

En base a nuevas prospecciones de campo realizadas en diversas localidades costeras de las regiones de Atacama y Coquimbo (Chile), entre los años 2006 y 2008, he podido observar y registrar una mayor amplitud trófica de algunas especies, lo cual implica que el conocimiento acerca de las interacciones que estas especies puedan tener en un sistema de desierto requiere de mayor profundización en el futuro. Si bien hasta la fecha estas especies habían sido descritas como fitófagas, la omnivoria en Gyriosomus

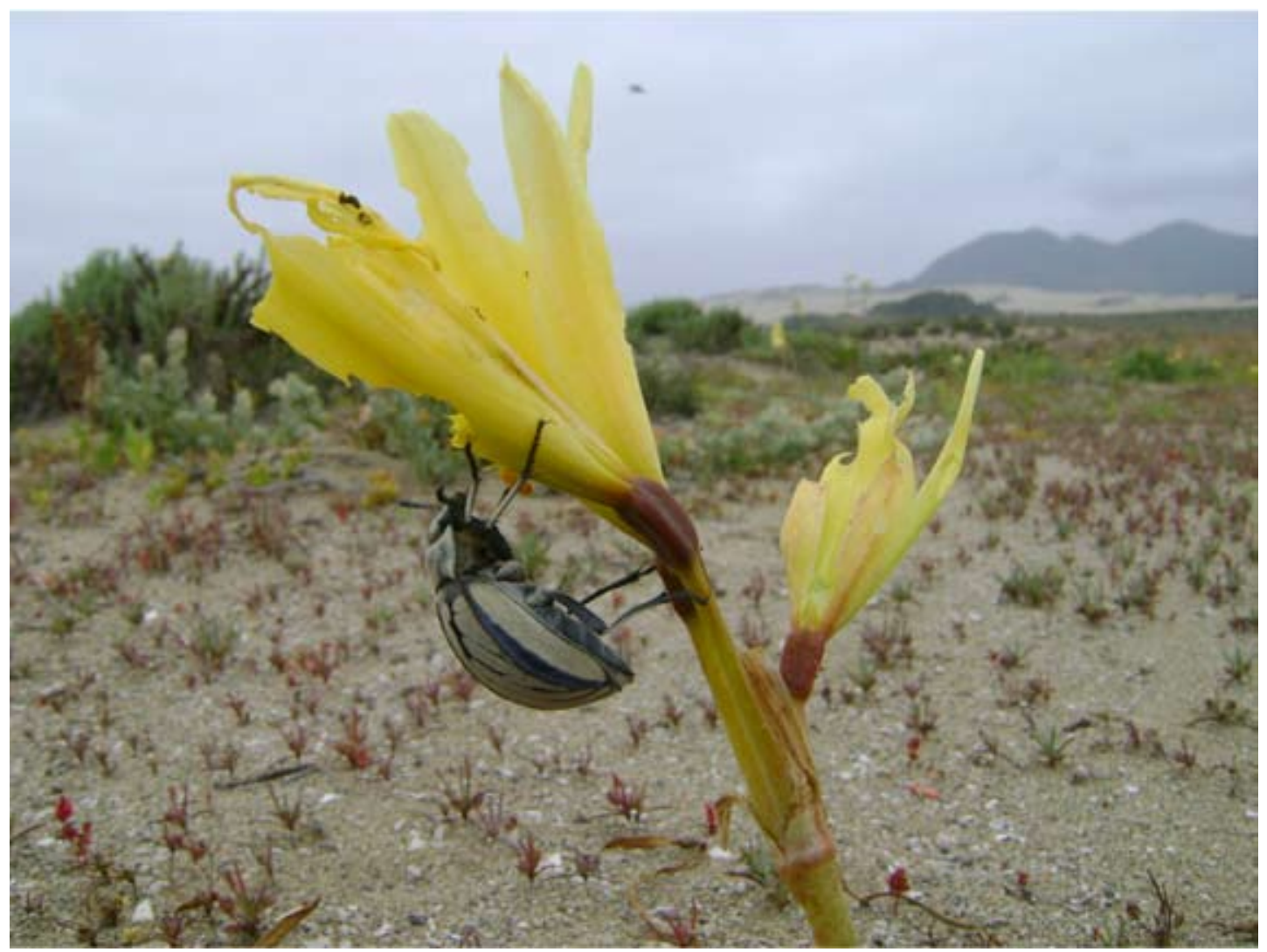

Figura 1. Gyriosomus elongatus (†) depredando pétalos de Rhodophiala bagnoldii (Liliales, Amaryllidaceae) (Punta de Choros, $29^{\circ} 21^{\prime} \mathrm{S}, 71^{\circ} 10^{\prime}$ O, Región de Coquimbo). 
barriai Kulzer, 1959, es elocuente. Esta especie se alimenta de detritos de origen vegetal o animal (Figura 2), pero he podido observar predación intraespecífica de exoesqueletos. La predación de otras especies también ocurre con Gyriosomus withei Waterhouse, 1844, que depreda larvas de Noctuidae (Lepidoptera: Heterocera) (Figura 3). Otro caso excepcional es el registro de individuos de Gyriosomus elongatus Waterhouse, 1843, depredando el cadáver de Liolaemus silvai Ortiz, 1989 (Reptilia, Tropiduridae) (Figura 4), ambas especies asociadas a paleodunas estabilizadas del sector de Punta de Choros (Región de Coquimbo, Chile).

Penry y Jumars (1987) han documentado que la selección del alimento está determinada fundamentalmente por su abundancia y disponibilidad, de modo que la selección por parte del depredador depende de la adquisición (Polis y Myers, 1989). Muchas de las estrategias de alimentación son fenómenos complejos que implican la obtención del alimento (reconocerlo, seleccionarlo y capturarlo) y su utilización (ingerirlo, digerirlo y excretarlo) (Krebs y Davis, 1997). En este sentido, las estrategias dietarias de las especies de Gyriosomus aquí registradas podrían depender de factores intrínsecos (i.e., fisiológicos) de cada especie. Por ejemplo, las especies de Gyriosomus muestran un marcado dimorfismo sexual, el cual puede modular la búsqueda y manipulación del alimento de acuerdo al potencial de calidad nutricional, más aún cuando esto ocurre en un sistema de desierto (Polis, 1991, 1993). Así, una hembra "preferiría" alimentarse de categorías de presa de mejor calidad, como los exoesqueletos o estadios preimaginales de otros artrópodos (Figura 3); esta estrategia se relacionaría con la inversión energética en la fase reproductiva que podría mejorar su capacidad de producción de huevos y ovipostura.

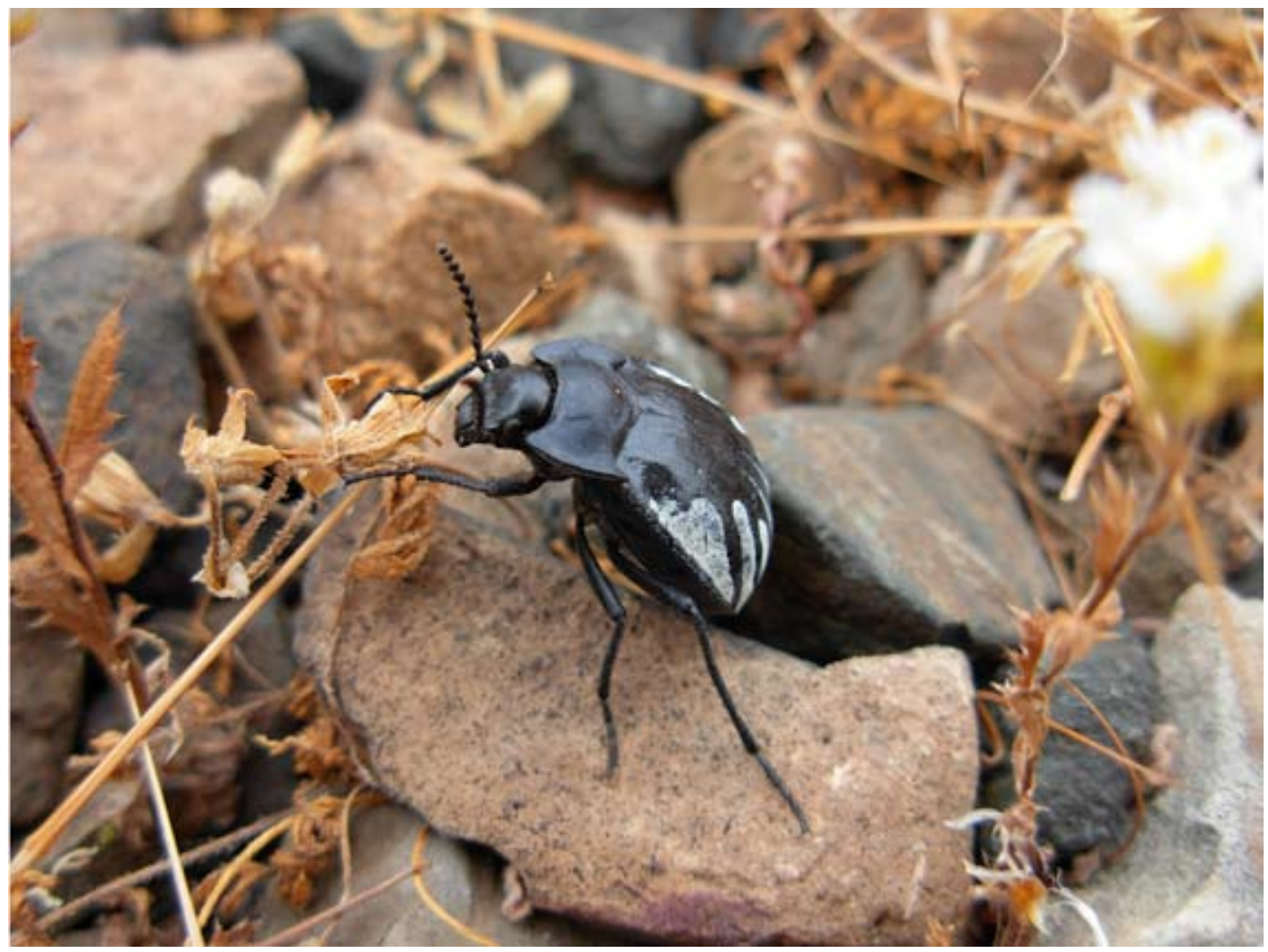

Figura 2. Gyriosomus barriai $\left({ }^{3}\right)$ alimentándose de detritos de origen vegetal (Los Choros, $29^{\circ} 17^{\prime}$ S, $71^{\circ} 18^{\prime}$ O, Región de Coquimbo). 
En ecosistemas áridos, la mayoría de los depredadores son arácnidos y reptiles, los cuales tienden a ser depredadores activos y generalistas (Polis, 1981; Punzo, 2000), y que son capaces de modular su tiempo de actividad para sincronizarlos con los tiempos de actividad de sus presas (Ayal, 2007). Sin embargo, las estrategias tróficas mostradas por Gyriosomus me hacen postular que este taxón está posicionado en niveles tróficos mayores, por lo que claramente se ha subestimado su capacidad de incidencia en la modulación de las actividades en estos ambientes. Probablemente, estas especies sean responsables de aumentar la productividad primaria y secundaria en estos ecosistemas (Oksanen et al., 1981), ya sea por su capacidad polinizadora aún desconocida o por su rol en la descomposición de elementos en el ambiente. Al igual como ocurre con Gyriosomus, es esperable que otros ensambles de tenebriónidos presenten variaciones en sus estrategias ecológicotróficas necesarias para optimizar el uso de los recursos disponibles en la fase húmeda (i.e., años

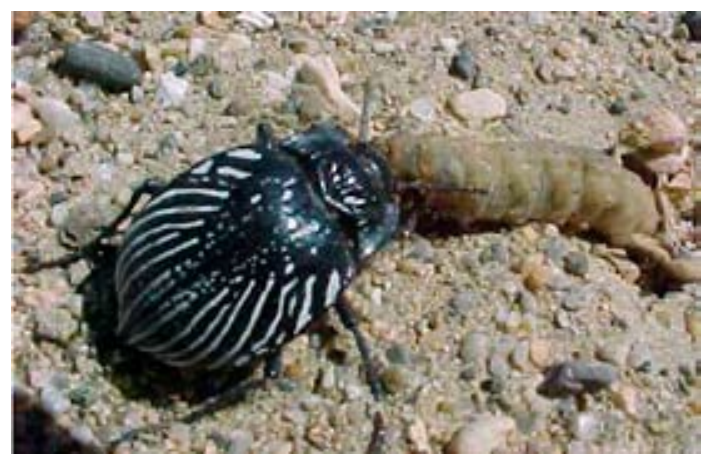

Figura 3. Gyriosomus withei (ㅇ) depredando una larva de Noctuidae (Lepidoptera: Heterocera) (Chañaral de Aceituno, $29^{\circ} 02^{\prime} \mathrm{S}, 71^{\circ} 25^{\prime} \mathrm{O}$, Región de Atacama).

húmedos no ENOS o años ENOS), esta vez más abundantes y de mejor calidad (Cepeda-Pizarro et al., 2005b). Las variaciones en la conducta de selección trófica de este grupo de Nycteliini plantean un conjunto de interrogantes respecto al rol funcional de estas especies en los ecosistemas áridos y semiáridos de Chile.

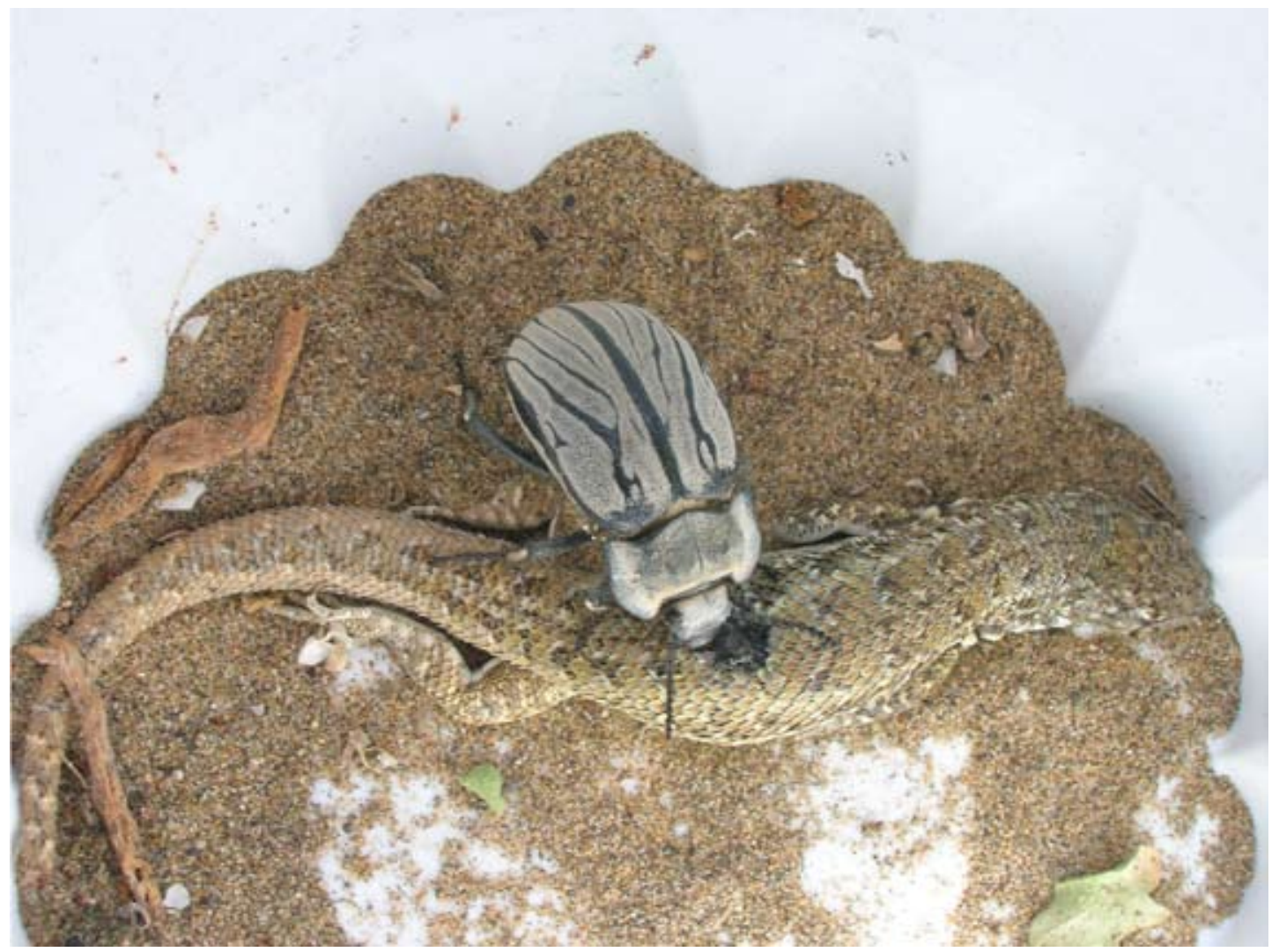

Figura 4. Gyriosomus elongatus (†) alimentándose de cadáver de Liolaemus silvai (Reptilia, Tropiduridae) (Punta de Choros, $29^{\circ} 21^{\prime} \mathrm{S}, 71^{\circ} 10^{\prime} \mathrm{O}$, Región de Coquimbo). 


\section{RECONOCIMIENTOS}

Agradezco a Marcela A. Vidal (Departamento de Zoología, Universidad de Concepción) y Gustavo E. Flores (IADIZA-CCT CONICET-Mendoza, Argentina) por la lectura crítica del manuscrito, y a

\section{LITERATURA CITADA}

ALFARO, F.M.; PIZARRO-ARAYA, J.; FLORES, G.E. (EN PRENSA) 2009. Epigean tenebrionids (Coleoptera: Tenebrionidae) from the Choros Archipelago (Coquimbo Region, Chile). Entomological News 120 (2): 125-130.

ARMESTO, J.J.; VIDIELLA, P.E.; GUTIÉRREZ, J.R. 1993. Plant communities of the fog-free coastal desert of Chile: plant strategies in a fluctuating environment. Revista Chilena de Historia Natural 66: 271-282.

AYAL, Y. 2007. Trophic structure and the role of predation in shaping hot desert communities. Journal of Arid Environments 68: 171-187.

CEPEDA-PIZARRO, J.; PIZARRO-ARAYA, J.; VÁSQUEZ, H. 2005a. Variación en la abundancia de Arthropoda en un transepto latitudinal del desierto costero transicional de Chile, con énfasis en los tenebriónidos epígeos. Revista Chilena de Historia Natural 78: 651-663.

CEPEDA-PIZARRO, J.; PIZARRO-ARAYA, J.; VÁSQUEZ, H. 2005b. Composición y abundancia de artrópodos del Parque Nacional Llanos de Challe: impactos del ENOS de 1997 y efectos del hábitat pedológico. Revista Chilena de Historia Natural 78: 635-650.

FLORES, G.E. 2000. Cladistic analysis of the Neotropical tribe Nycteliini (Coleoptera: Tenebrionidae). Journal of the New York Entomological Society 108: 13-25.

GAJARDO, R. 1993. La vegetación natural de Chile. Editorial Universitaria, Santiago, Chile. 165 pp.

GEBIEN, H. 1944. Die Gattung Gyriosomus Guér. Coleoptera, Tenebrionidae. Mitteilungen der Münchener Entomologischen Gesellschaft 34: 135-194.

KREBS, J.R.; DAVIS, N.B. I997. Behavioral ecology. An evolutionary approach. 4th ed. Blackwell Scientific, London. 464 pp.

MONDACA, J. 2004. Nueva especie de Gyriosomus GuérinMéneville, 1834 (Coleoptera: Tenebrionidae: Nycteliini) del extremo norte de la Región de Atacama (Chile). Revista Chilena de Entomología 30: 21-26.

MORRONE, J.J. 2006. Biogeographic areas and transition zones of Latin America and the Caribbean islands based on panbiogeographic and cladistic analyses of the entomofauna. Annual Review of Entomology 51: 467-494.

OKSANEN, L.; FRETWELL, S.D.; ARRUDA, J.; NIEMELA, P. 1981. Exploitation ecosystems in gradients of primary productivity. American Naturalist 118: 240-261.

PEÑA, L.E. 1959. Las vaquitas del desierto. Noticiero Mensual del Museo Nacional de Historia Natural (Chile) 37: 2.
Marcela A. Vidal y Juan Carlos Ortiz (Departamento de Zoología, Universidad de Concepción) por la determinación del tropidúrido. Trabajo financiado por el proyecto DIULS-PF07101 de la Universidad de La Serena, Chile.

PENRY, D.L.; JUMARS, P.A. 1987. Modelling animal guts as chemical reactors. American Naturalist 129: 69-96.

PIZARRO-ARAYA, J.; FLORES, G.E. 2004. Two new species of Gyriosomus Guérin-Méneville from the Chilean coastal desert (Coleoptera: Tenebrionidae: Nycteliini). Journal of the New York Entomological Society, 112: 121-126.

PIZARRO-ARAYA, J.; FLORES, G.E. 2006. La posición sistemática de Geoborus lineatus (Guérin-Méneville), comb. nov. (ex. Gyriosomus) (Coleoptera: Tenebrionidae). Revista de la Sociedad Entomológica Argentina 65: 85-90.

PIZARRO-ARAYA, J.; JEREZ, V. 2004. Distribución geográfica del género Gyriosomus Guérin-Méneville, 1834 (Coleoptera: Tenebrionidae): una aproximación biogeográfica. Revista Chilena de Historia Natural 77: 491-500.

PIZARRO-ARAYA, J.; CEPEDA-PIZARRO, J.; FLORES, G.E. 2008. Diversidad taxonómica de los artrópodos epígeos de la Región de Atacama (Chile): estado del conocimiento. En: Squeo, F.A., G. Arancio y J.R. Gutiérrez (eds.). Libro Rojo de la Flora Nativa y de los Sitios Prioritarios para su Conservación: Región de Atacama: 257-274. Ediciones Universidad de La Serena, La Serena. Chile. xvi + 456 pp.

POLIS, G.A. 1981. The evolution and dynamics of intraspecific predation. Annual Review of Ecology and Systematics 12: $125-151$.

POLIS, G.A. 1991. Complex trophic interactions in deserts -an empirical critique of food-web theory. American Naturalist 138: $123-155$.

POLIS, G.A. (1993). Scorpions as model vehicles to advance theories of population and community ecology: the role of scorpions in desert communities. Memoirs of the Queensland Museum 33: 401-410.

POLIS, G.A.; A. MYERS (1989). The ecology and evolution of intraguild predation: potential competitors that eat each other. Annual Review of Ecology and Systematics 20: 297-330.

PUNZO, F. (2000). Desert arthropods: life history variations. Springer, Heidelberg, $308 \mathrm{pp}$.

SÁIZ, F.; SOLERVICENS, J.; OJEDA, P. 1989. Coleópteros del Parque Nacional La Campana y Chile Central. Ediciones Universitarias de Valparaíso, Chile. 124 pp.

VIDAL, P.; GUERRERO, M. 2007. Los Tenebrionidae de Chile. Ediciones Universidad Católica de Chile. Santiago, Chile. 478 pp. 
